\title{
Description of $x$-ray reflection and diffraction from periodical multilayers and superlattices by the eigenwave method
}

\author{
I. D. Feranchuk, ${ }^{1}$ S. I. Feranchuk, ${ }^{1}$ A. A. Minkevich, ${ }^{1}$ and A. Ulyanenkov ${ }^{2}$ \\ ${ }^{1}$ Byelorussian State University, Francisk Skariny Av., 4, 220050 Minsk, Belarus \\ ${ }^{2}$ Bruker AXS, Östliche Rheinbrückenstr. 49, 76187 Karlsruhe, Germany
}

(Received 12 May 2003; published 8 December 2003)

\begin{abstract}
The analytical solution of recurrent equations for amplitudes of electromagnetic field is found for description of x-ray reflection and diffraction from periodical multilayered media. The method proposed uses the Bloch eigenwaves approach for periodical structure, which reduces considerably the computer time required for simulation of diffracted/reflected x-ray intensity and, therefore, accelerates the fitting trial-and-error procedure for sample model parameters. Numerical examples and fit results for experimental $\mathrm{x}$-ray data are provided to demonstrate the effectiveness of method. A new parameter describing the fluctuation of superlattice period is introduced and its influence on experimental data interpretation is discussed.
\end{abstract}

DOI: 10.1103/PhysRevB.68.235307

PACS number(s): 68.65.Cd, 61.10.Kw

\section{INTRODUCTION}

In recent decade, a numerous analytical methods and techniques have been developed and reported for calculation of x-ray reflectivity, diffraction, and diffuse scattering spectra (see, for example Ref. 1). However, increasing demand for the x-ray methods from industry and science emphasizes the problem of algorithm improvements, both in acceleration and precision aspects. This task is especially important when the experimental data from complex samples have to be fastly and accurately fitted by theoretical models.

The multilayers and superlattices, consisting of large number of repeating periods of basic thin layers, make up a wide class of samples in semiconductor and nanotechnology industries. For investigation of these structures x-ray methods are proved to be very advantageous. In general case, the solution of Maxwell's equations for x-ray wave field in multilayers is reduced to the system of recurrent equations, the scalar ones for reflectivity, ${ }^{2}$ and matrix ones for diffraction. ${ }^{3}$ The theoretical interpretation of experimental $x$-ray data, based on the transfer matrices, ${ }^{3}$ delivers convenient formalism to solve general recurrent equations for multilayered structures in both reflectivity ${ }^{2}$ and diffraction ${ }^{4}$ cases. For periodical structures, using the powers of the transfer matrix for basic element of superlattice (SL), the time of calculation can be considerably reduced. ${ }^{5}$ However, this technique requires the calculation of high powers of matrices, which is also time-consuming procedure because of the number of numerical operations exponentially increases with the number of SL elements. There are several approximated methods for reduction of calculation time, for example, kinematical approach and single-reflection approximation, ${ }^{1}$ but they do not provide sufficient precision for thick SL's and superlattices with large number of layers. Thus, the development of methods reducing the calculation time for x-ray reflectivity and diffraction from periodical multilayered structures is an actual task of applied x-ray physics.

The method proposed in this paper utilizes the possibility to express the Bloch eigenwaves of one-dimensional periodical infinite layer stack through the solutions of x-ray scatter- ing problem within the single basic element composing the superlattice period. ${ }^{6}$ For superlattice with limited number of periods these Bloch eigenwaves can further be used along with the boundary conditions for whole layer stack. This results in analytical expression for the electromagnetic field and integral reflection coefficient in any point of sample, with no need to solve the recurrent equations. This approach is further called in this paper a "method of eigenwaves" (MEW). In the present paper, the effectiveness of MEW is analyzed for calculation of $\mathrm{x}$-ray reflectivity (XRR) and high-resolutions $\mathrm{x}$-ray diffraction (HRXRD) intensities from superlattices. Comparison of MEW with other approaches demonstrates the essential time reduction for calculation and fitting of x-ray intensities. The paper is arranged as follows. In Sec. II the analytical solution for reflection coefficient from bilayered periodical superlattice is found. The results of calculations coincide with the numerical solution of the same problem by Parratt's equations, however, the advantage of MEW is the considerable acceleration of the simulation process. In Sec. III, the superlattice with arbitrary basic periodical element is considered. The combination of Parratt's recursive equations with MEW is used to achieve the best performance of simulation technique. We discuss also in this section a possible application of obtained solutions for simulation and fitting of diffuse x-ray scattering from superlattices with rough interfaces. A parameter for characterization of superlattice period fluctuation is also introduced to obtain a closer fit of theory and experiment. In Sec. IV, MEW approach is extended to description of $\mathrm{x}$-ray diffraction from superlattices. The diffraction process inside basic periodical element of superlattice is calculated by matrix method, ${ }^{4}$ and the evolution of diffraction into entire superlattice is described by MEW.

\section{MEW FOR X-RAY REFLECTIVITY FROM TWO- COMPONENT SUPERLATTICE}

Let us consider the reflection of monochromatic $\mathrm{x}$-ray beam with certain polarization and wave number $k=2 \pi / \lambda$ from a multilayered structure consisting of $N$ layers grown on substrate. The structure is assumed to be a superlattice 


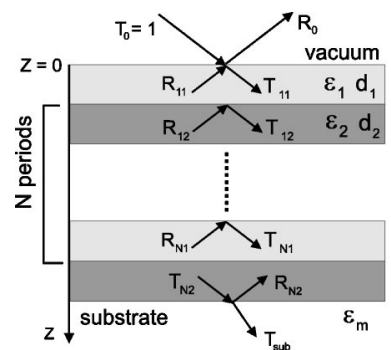

a

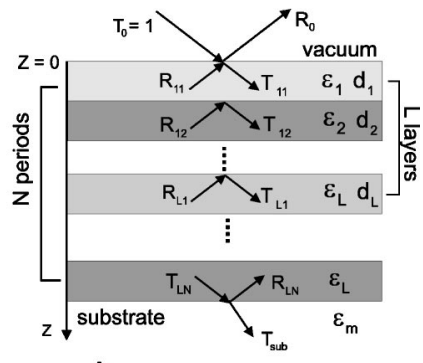

b
FIG. 1. Sketch of the wave fields for (a) bilayered and (b) $L$-layered superlattices.

composed of multiple repetition of basic layer period, consisting of two layers [see Fig. 1(a)] and the $\mathrm{x}$ rays impinge the sample at the incidence angle $\alpha$. In x-ray reflectometry, the lateral dimension of a layer is supposed to be infinite, which results in (i) conservation of lateral component of wave vector, and (ii) dependence of wave field on solely $z$ component of wave vector. For the problem considered in this paper, the following variables, defined through the permittivity $\epsilon$, are essential for description of scattering process

$$
\begin{gathered}
k_{z 0}=k \sin \alpha, \quad k_{z m}=k \sqrt{\epsilon_{m}-\cos ^{2} \alpha}, \\
k_{z 1}=k \sqrt{\epsilon_{1}-\cos ^{2} \alpha}, \quad k_{z 2}=k \sqrt{\epsilon_{2}-\cos ^{2} \alpha}, \\
\epsilon_{t}=n_{t}^{2}=\left(1-\delta_{t}+i \beta_{t}\right)^{2},
\end{gathered}
$$

where $z$ axis is a perpendicular inward normal to the sample surface; the values $1-\delta_{t}$ and $\beta_{t}$ are the real and imaginary components $^{7}$ of refractive index $n_{t}, t=0, m, 1,2$ for vacuum, substrate, and layers, respectively. Let us denote by $d_{1}$ and $d_{2}$ the thicknesses of the layers, then $d=d_{1}+d_{2}$ is the superlattice period. The general solution of Maxwell's equations for two layers in the period with number $l(l=0$ corresponds to vacuum) is

$$
\begin{gathered}
E_{l}(x, z)=e^{i k x \cos \alpha} \Psi_{l}(z) ; \\
\Psi_{l}(z)=T_{1 l} e^{i k_{z 1}(z-l d)}+R_{1 l} e^{-i k_{z 1}(z-l d)}, \\
l d \leqslant z \leqslant l d+d_{1}, \\
\Psi_{l}(z)=T_{2 l} e^{i k_{z 2}(z-l d)}+R_{2 l} e^{-i k_{z 2}(z-l d)}, \\
l d+d_{1} \leqslant z \leqslant(l+1) d .
\end{gathered}
$$

The condition of wave field continuity at the boundary between the layers 1 and 2 within the $l$ period of SL

$$
\begin{gathered}
T_{1 l} e^{i k_{z 1} d_{1}}+R_{1 l} e^{-i k_{z 1} d_{1}}=T_{2 l} e^{i k_{z 2} d_{1}}+R_{2 l} e^{-i k_{z 2} d_{1}}, \\
k_{z 1}\left[T_{1 l} e^{i k_{z 1} d_{1}}-R_{1 l} e^{-i k_{z 1} d_{1}}\right]=k_{z 2}\left[T_{2 l} e^{i k_{z 2} d_{1}}-R_{2 l} e^{-i k_{z 2} d_{1}}\right]
\end{gathered}
$$

is used to express the reflection and transmission coefficients in second layer via the corresponding parameters of the first layer:

$$
\begin{gathered}
T_{2 l}=\beta_{11} T_{1 l}+\beta_{12} R_{1 l}, \quad R_{2 l}=\beta_{21} T_{1 l}+\beta_{22} R_{1 l}, \\
\beta_{11}=\frac{k_{z 2}+k_{z 1}}{2 k_{z 2}} e^{i\left(k_{z 1}-k_{z 2}\right) d_{1}}, \quad \beta_{12}=\frac{k_{z 2}-k_{z 1}}{2 k_{z 2}} e^{-i\left(k_{z 1}+k_{z 2}\right) d_{1}}, \\
\beta_{21}=\frac{k_{z 2}-k_{z 1}}{2 k_{z 2}} e^{i\left(k_{z 1}+k_{z 2}\right) d_{1}}, \quad \beta_{22}=\frac{k_{z 2}+k_{z 1}}{2 k_{z 2}} e^{-i\left(k_{z 1}-k_{z 2}\right) d_{1}} .
\end{gathered}
$$

The same conditions at the boundary between the layers $l$ and $l+1$ result in

$$
\begin{array}{cc}
T_{1(l+1)}=\gamma_{11} T_{2 l}+\gamma_{12} R_{2 l}, & R_{1(l+1)}=\gamma_{21} T_{2 l}+\gamma_{22} R_{2 l}, \\
\gamma_{11}=\frac{k_{z 2}+k_{z 1}}{2 k_{z 1}} e^{i k_{z 2} d}, & \gamma_{12}=\frac{k_{z 1}-k_{z 2}}{2 k_{z 1}} e^{-i k_{z 2} d}, \\
\gamma_{21}=\frac{k_{z 1}-k_{z 2}}{2 k_{z 1}} e^{i k_{z 2} d}, & \gamma_{22}=\frac{k_{z 2}+k_{z 1}}{2 k_{z 1}} e^{-i k_{z 2} d} .
\end{array}
$$

To make an expression more clear, the $(2 \times 2)$ transfer matrix $\hat{M}$ can be introduced, ${ }^{3}$ which defines the transformation of transmission and reflection coefficients by a single layer of the basic period of superlattice

$$
\begin{array}{cl}
T_{1(l+1)}=M_{11} T_{1 l}+M_{12} R_{1 l}, & R_{1(l+1)}=M_{21} T_{1 l}+M_{22} R_{1 l}, \\
M_{11}=\beta_{11} \gamma_{11}+\beta_{21} \gamma_{12}, & M_{12}=\beta_{12} \gamma_{11}+\beta_{22} \gamma_{12}, \\
M_{21}=\beta_{11} \gamma_{21}+\beta_{21} \gamma_{22}, & M_{22}=\beta_{12} \gamma_{21}+\beta_{22} \gamma_{22} .
\end{array}
$$

If the phase of wave field is defined in accordance with Eq. (2), then the transfer matrix does not depend on index $l$ numbering the superlattice layers. This fact makes it possible to use the powers of matrix $\hat{M}$ for calculation of full transfer matrix of superlattice. ${ }^{5}$ Such an approach reduces the calculation time in comparison with the direct solution of recurrent equations. However, MEW simplifies this solution even more because it expresses the total reflection coefficient in analytical form. To derive this form, the two-component eigenvectors $\mathbf{A}^{(s)}=\left(T^{(s)}, R^{(s)}\right)$; $s=1,2$ of matrix $\hat{M}$ has to be introduced

$$
\begin{gathered}
\hat{M} \mathbf{A}^{(s)}=\lambda_{s} \mathbf{A}^{(s)}, \\
\lambda_{1,2}=\frac{M_{11}+M_{22}}{2} \pm \sqrt{\frac{\left(M_{11}-M_{22}\right)^{2}}{4}+M_{12} M_{21}} \\
R^{(s)}=\nu_{s} T^{(s)}=\frac{\lambda_{s}-M_{11}}{M_{12}} T^{(s)} .
\end{gathered}
$$

The wave fields determined by coefficients (7) create a basis of eigenwaves $(\mathrm{EW})$ in infinite periodical layer stack. If the eigenvalues are indexed in such a way that $\left|\lambda_{1}\right|<\left|\lambda_{2}\right|$, the values $T^{(s)}$ then represent the amplitudes of EW, which are excited in the finite stack by the incident plane wave. To find these amplitudes $T^{(s)}$, the boundary conditions have to be 
used at the interfaces superlattice/vacuum and superlattice/ substrate. The wave field at first interface is represented as a superposition of eigenwaves:

$$
\Psi_{1}(z)=T^{(1)}\left[e^{i k_{1} z}+\nu_{1} e^{-i k_{1 z^{z}}}\right]+T^{(2)}\left[e^{i k_{1} z}+\nu_{2} e^{-i k_{1 z^{z}}}\right] .
$$

The continuity of this wave field at the surface $z=0$ results in equations

$$
\begin{gathered}
1+R_{0}(\alpha)=T^{(1)}\left(1+\nu_{1}\right)+T^{(2)}\left(1+\nu_{2}\right), \\
k_{z 0}\left[1-R_{0}(\alpha)\right]=k_{z 1}\left[T^{(1)}\left(1-\nu_{1}\right)+T^{(2)}\left(1-\nu_{2}\right)\right],
\end{gathered}
$$

where $R_{0}(\alpha)$ is the integral reflection coefficient from the entire superlattice. Because of Eq. (7) for EW, the reflection and transmission coefficients in the last layer with number $N$ are

$$
\begin{gathered}
T_{1 N}=\lambda_{1}^{N} T^{(1)}+\lambda_{2}^{N} T^{(2)}, \quad R_{1 N}=\nu_{1} \lambda_{1}^{N} T^{(1)}+\nu_{2} \lambda_{2}^{N} T^{(2)}, \\
T_{2 N}=\beta_{11} T_{1 N}+\beta_{12} R_{1 N}, \quad R_{2 N}=\beta_{21} T_{1 N}+\beta_{22} R_{1 N} .
\end{gathered}
$$

The boundary conditions at interface $z=N d$, where wave field is only defined by transmission coefficient $T_{s u b}$, complete the equations system for four unknown variables $R_{0}$, $T^{(1)}, T^{(2)}$, and $T_{\text {sub }}$

$$
\begin{gathered}
T_{s u b} e^{i k_{z s} N d}=T_{(2 N)} e^{i k_{z 2} d}+R_{(2 N)} e^{-i k_{z 2} d}, \\
k_{z m} T_{s u b} e^{i k_{z m} N d}=k_{z 2}\left[T_{(2 N)} e^{i k_{z 2} d}-R_{(2 N)} e^{-i k_{z 2} d}\right] .
\end{gathered}
$$

The equations system [(9)-(11)] delivers the expression for $R_{0}(\alpha)$, which is, in fact, the analytical solution of the recurrent Parratt's equations for the whole stack of the layers

$$
\begin{gathered}
R_{0}(\alpha)=-\frac{k_{z 1}\left[1-\nu_{1}+P_{N}\left(1-\nu_{2}\right)\right]-k_{z 0}\left[1+\nu_{1}+P_{N}\left(1+\nu_{2}\right)\right]}{k_{z 1}\left[1-\nu_{1}+P_{N}\left(1-\nu_{2}\right)\right]+k_{z 0}\left[1+\nu_{1}+P_{N}\left(1+\nu_{2}\right)\right]}, \\
P_{N}=-\left(\frac{\lambda_{1}}{\lambda_{2}}\right)^{N} \frac{\left(k_{z m}-k_{z 2}\right)\left(\beta_{11}+\beta_{12} \nu_{1}\right) e^{i k_{z 2} d}+\left(k_{z m}+k_{z 2}\right)\left(\beta_{21}+\beta_{22} \nu_{1}\right) e^{-i k_{z 2} d}}{\left(k_{z m}-k_{z 2}\right)\left(\beta_{11}+\beta_{12} \nu_{2}\right) e^{i k_{z 2} d}+\left(k_{z m}+k_{z 2}\right)\left(\beta_{21}+\beta_{22} \nu_{2}\right) e^{-i k_{z 2} d}}
\end{gathered}
$$

Figure 2 shows simulated by formula (12) x-ray reflectivity from $(\mathrm{Si} / \mathrm{Ge})_{60}$ superlattice with the thickness of layers $d_{1}=10 \mathrm{~nm}, d_{2}=20 \mathrm{~nm}$ on Si substrate. The curve is identical to the one calculated by recurrent Parratt's equations. ${ }^{2}$ However, the computer time required for these both simulations is essentially different, especially when the number of superlattice periods is large (see inset in Fig. 2). The numeri-

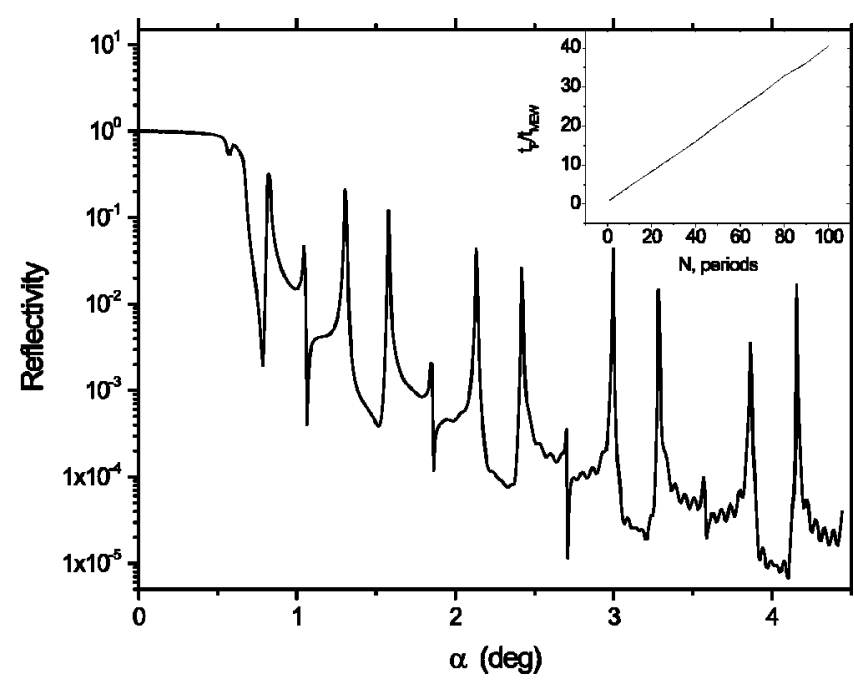

FIG. 2. X-ray reflectivity from $(\mathrm{Si} / \mathrm{Ge})_{60}$ superlattice on the $\mathrm{Si}$ substrate simulated both by Parratt's equations and MEW. Inset shows the ratio of the computer times required for simulations by Parratt's equations $\left(t_{P}\right)$ and $\mathrm{MEW}\left(t_{M E W}\right)$ as a function SL periods number. cal solution of the Parratt's equations ${ }^{2}$ involves $\simeq 4(4)^{N}$ operations, which increases the simulation time exponentially. When the powers of matrices are used for calculation of $R_{0}(\alpha)$, the time increases as a power law with increasing $N$ Ref. 5, whereas the use of Eq. (12) makes the number of operations to be independent of $N$. This advantage of MEW is even more pronounced for the experimental data fitting routines, which simulate the x-ray reflectivity many times during the trial-and-error procedure.

\section{MEW FOR X-RAY REFLECTIVITY FROM SUPERLATTICE WITH ARBITRARY STRUCTURE OF THE BASIC PERIOD}

The technique presented in preceeding section can be also applied to complicated sample models, for example, to the superlattice, the basic period of which consists of $L$ layers with thicknesses $d_{j}, j=1, \ldots, L$; so that the total basic period is

$$
\sum_{j} d_{j}=d,
$$

and refraction indices $n_{j}$ [Fig. 1(b)]. This kind of sample model describes both the superlattices with basic period containing more than two layers and bilayer superlattices with intermediate graded interfaces, which yield in this case parasitic artificial interlayers. For these samples, the combined technique can be used: numerical solution for recurrent equations with $(2 \times 2)$ transfer matrix $\hat{M}_{L}$ for basic period is 
supplemented with MEW approach for analytical calculation of total reflection coefficient $R_{0}(\alpha)$.

The wave field within the $j$ layer of the first basic period is defined by Eq. (2) with coefficients $T_{j}, R_{j}$ following from the equations ${ }^{2}$ [the sketch of wave fields in sublayers of the basic period is shown in Fig. 1(b)]

$$
\begin{aligned}
& T_{1, j+1}=\frac{1}{t_{j+1, j}}\left[T_{1, j} e^{i\left(k_{z(j+1)}-k_{z j}\right) z_{j}}+R_{1, j} r_{j+1, j} e^{i\left(k_{z(j+1)}+k_{z j}\right) z_{j}}\right], \\
& R_{1, j+1}=\frac{1}{t_{j+1, j}}\left[T_{1, j} r_{j+1, j} e^{-i\left(k_{z(j+1)}+k_{z j}\right) z_{j}}\right. \\
& +R_{1, j} e^{\left.-i\left(k_{z(j+1)}-k_{z j}\right) z_{j}\right]}, \\
& t_{j+1, j}=\frac{2 k_{z j}}{k_{z j}+k_{z(j+1)}}, \quad r_{j+1, j}=\frac{k_{z j}-k_{z(j+1)}}{k_{z j}+k_{z(j+1)}}, \\
& k_{z, j}=k \sqrt{n_{j}^{2}-\cos ^{2} \alpha} .
\end{aligned}
$$

To find the eigenwaves of the system, the transfer matrix $\hat{M}_{L}$ has to be calculated for basic period. By the definition, this matrix gives a relation between the coefficients $T_{1,(L+1)}$ $=T_{2,1}, R_{1,(L+1)}=T_{2,1}$ and parameters $T_{1}, R_{1}$ for wave fields $\Psi_{j}$ inside the superlattice. The formal solution of this problem can be found by successive iterative solution of Eqs. (13), and the result is presented in compact matrix form ${ }^{1}$

$$
\begin{gathered}
\Psi_{1,(L+1)}=\Psi_{2,1}=\hat{\Phi}_{L} \hat{Q}_{L}^{-1} \hat{\Phi}_{L-1} \hat{Q}_{L-1}^{-1} \ldots \hat{\Phi}_{2} \hat{Q}_{2}^{-1} \hat{\Phi}_{1} \Psi_{1,1}, \\
Q_{j}=\frac{1}{t_{j+1, j}}\left(\begin{array}{cc}
1 & r_{j+1, j} \\
r_{j+1, j} & 1
\end{array}\right), \quad \Phi_{j}=\left(\begin{array}{cc}
e^{-i k_{z j} z_{j}} & 0 \\
0 & e^{i k_{z j} z_{j}}
\end{array}\right) .
\end{gathered}
$$

Despite of formal simplicity of this solution, the real calculation of matrix $\hat{M}$ involves the calculation of the products of $2 L(2 \times 2)$ matrices, i.e., $\simeq 4(8)^{L}$ operations. The more effective algorithm is based on scalar Parratt's equations, ${ }^{2}$ which reduces the operations number down to $\simeq 4(4)^{L}$

$$
X_{j}=\frac{R_{j}}{T_{j}}=e^{-2 i k_{z j}} \frac{r_{j, j+1}+X_{j+1} e^{2 i k_{z(j+1)} z_{j}}}{1+r_{j, j+1} X_{j} e^{2 i k_{z(j+1) z_{j}}}} .
$$

To use these equations for calculation of matrix $\hat{M}$, the boundary conditions for $X_{j}$ have to be changed. In reflectometry, the boundary conditions $X_{L}=0, R_{L}=0, T_{0}=1$ are usually used for the structure containing $L$ layers. However, in the considered case here the initial transmission and reflection coefficients are not directly related. The most convenient way to calculate the transition matrix $\hat{M}$ seems to be above-mentioned matrix method. If phases of the wave fields in the first layer of basic period are defined as in Eq. (2), then matrix $\hat{M}$ depends no longer on the repetition number of basic periods, and Eq. (14) is modified. The recurrent equations (13) being resulted from the transformation of vectors $\left(T_{j}, R_{j}\right)$ are represented then by matrices

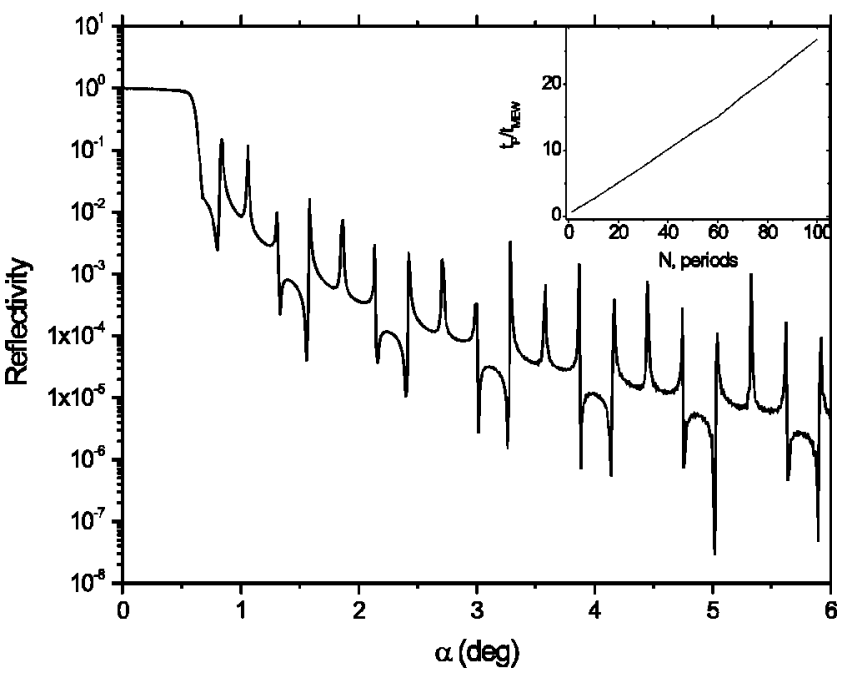

FIG. 3. Simulated X-ray reflectivity from (AlAs/GaAs/InAs/GaSb) ${ }_{40}$ superlattice on the GaAs substrate. Insert shows the ratio of the computer times required for simulation by Parratt's equations $\left(t_{P}\right)$ and $\mathrm{MEW}\left(t_{M E W}\right)$ as the function of SL periods number.

$$
\begin{gathered}
\hat{A}_{j}=\frac{1}{t_{j+1, j}}\left(\begin{array}{cc}
e^{i\left(k_{z j}-k_{z(j+1)}\right) z_{j}} & r_{j+1, j} e^{-i\left(k_{z j}+k_{z(j+1)}\right) z_{j}} \\
r_{j+1, j} e^{i\left(k_{z j}+k_{z(j+1)}\right) z_{j}} & e^{-i\left(k_{z j}-k_{z(j+1)}\right) z_{j}}
\end{array}\right), \\
\hat{B}_{j}=\frac{1}{t_{j+1, j}}\left(\begin{array}{cc}
e^{i k_{z j} z_{j}} & r_{j+1, j} e^{-i k_{z j} z_{j}} \\
r_{j+1, j} e^{i k_{z j} z_{j}} & e^{-i k_{z j}}
\end{array}\right)
\end{gathered}
$$

The matrices $\hat{B}_{j}$ have to be used at both interfaces of basic period to eliminate the phase in the matrix $\hat{M}$ at the interface between the basic periods. Finally, the resulting transition matrix is

$$
\hat{M}=\hat{B}_{L} \hat{A}_{L-1} \hat{A}_{L-2} \ldots \hat{A}_{2} \hat{B}_{1} .
$$

To satisfy the continuity conditions (11) for wave fields at the interface between bottommost basic layer and substrate, the amplitudes $T_{N, L}$ and $R_{N, L}$ have to be expressed through the amplitudes $T_{N, 1}$ and $R_{N, 1}$ by means of the matrix

$$
\hat{M}_{L-1}=\hat{A}_{L-1} \hat{A}_{L-2} \ldots \hat{A}_{2} \hat{B}_{1} .
$$

Further calculations are analogous to two-component case, described in the preceeding section. In particular, the eigenvalues $\lambda_{1,2}$ and their eigenvectors follow from Eqs. (6) and (7), if the elements of the matrix $\hat{M}$ in Eq. (17) are used. The equations for amplitudes resulting from the boundary conditions for wave fields at the surface of superlattice and at the interface between superlattice and substrate are then written as

$$
\begin{aligned}
1+R_{0}(\alpha)=T_{1,1} & +R_{1,1}=T^{(1)}\left(1+\nu_{1}\right)+T^{(2)}\left(1+\nu_{2}\right), \\
k_{z 0}\left[1-R_{0}(\alpha)\right] & =k_{z 1}\left[T_{1,1}-R_{1,1}\right] \\
& =k_{z 1}\left[T^{(1)}\left(1-\nu_{1}\right)+T^{(2)}\left(1-\nu_{2}\right)\right],
\end{aligned}
$$




$$
\begin{gathered}
T_{\text {sub }} e^{i k_{z s} N d}=T_{N, L} e^{i k_{z L} d}+R_{N, L} e^{-i k_{z L} d}, \\
k_{z m} T_{s u b} e^{i k_{z m} N d}=k_{z L}\left[T_{N, L} e^{i k_{z L} d}-R_{N, L} e^{-i k_{z L} d}\right] .
\end{gathered}
$$

The amplitudes within the arbitrary $n$ period of SL are expressed through the amplitudes inside the first period by the following analytical formulas

$$
T_{n, 1}=\lambda_{1}^{(n-1)} T^{(1)}+\lambda_{2}^{(n-1)} T^{2)},
$$

$$
\begin{gathered}
R_{n, 1}=\nu_{1} \lambda_{1}^{(n-1)} T^{(1)}+\nu_{2} \lambda_{2}^{(n-1)} T^{(2)}, \\
R_{n, L}^{(1,2)}=\left(\hat{M}_{L-1}\right)_{21} T_{n, 1}+\nu_{(1,2)}\left(\hat{M}_{L-1}\right)_{22} R_{n, 1}, \\
T_{n, L}^{(1,2)}=\left(\hat{M}_{L-1}\right)_{11} T_{n, 1}+\nu_{(1,2)}\left(\hat{M}_{L-1}\right)_{12} R_{n, 1},
\end{gathered}
$$

where $n$ is assumed to be equal to $N$ when the amplitudes are substituted in Eq. (20). The formula (12) for parameter $P_{N}$ is then slightly transformed to

$$
\begin{gathered}
R_{0}(\alpha)=-\frac{k_{z 1}\left[1-\nu_{1}+P_{N}\left(1-\nu_{2}\right)\right]-k_{z 0}\left[1+\nu_{1}+P_{N}\left(1+\nu_{2}\right)\right]}{k_{z 1}\left[1-\nu_{1}+P_{N}\left(1-\nu_{2}\right)\right]+k_{z 0}\left[1+\nu_{1}+P_{N}\left(1+\nu_{2}\right)\right]}, \\
P_{N}=-\left(\frac{\lambda_{1}}{\lambda_{2}}\right)^{N} \frac{\left(k_{z m}-k_{z L}\right)\left(T_{L}^{(1)} e^{i k_{z L} d}+\left(k_{z m}+k_{z L}\right) R_{L}^{(1)} e^{-i k_{z L} d}\right.}{\left(k_{z m}-k_{z L}\right) T_{L}^{(2)} e^{i k_{z L} d}+\left(k_{z m}+k_{z L}\right) R_{L}^{(2)} e^{-i k_{z L} d}}, \\
R_{L}^{(1,2)}=\left(\hat{M}_{L-1}\right)_{21}+\nu_{(1,2)}\left(\hat{M}_{L-1}\right)_{22}, \quad T_{L}^{(1,2)}=\left(\hat{M}_{L-1}\right)_{11}+\nu_{(1,2)}\left(\hat{M}_{L-1}\right)_{12} .
\end{gathered}
$$

Figure 3 demonstrates the simulated $x$-ray reflectivity curves from the multicomponent superlattice $(\mathrm{AlAs} / \mathrm{GaAs} / \mathrm{InAs} / \mathrm{GaSb})_{40}$ on the GaAs substrate, where the layer thicknesses are equal to $(10 / 20 / 5 / 10)_{40} \mathrm{~nm}$, respectively. The comparison of required computer time for both straightforward Parratt's approach $t_{P}$ and MEW technique $t_{M}$ as the function of repetition period is presented in the inset in Fig. 3. In some cases the calculation time is reduced from several seconds to the fraction of seconds for single simulation by using MEW. At first sight, this reduction seems to be not in principal for real applications because absolute computation time is relatively short. However, in most cases, the treatment of x-ray experimental data from real superlattices requires the fitting of many parameters (thickness, roughness, electron density, lattice mismatches, etc.), which often are approximately known from growth conditions. Moreover, possible aperiodicity of SL structure and large number of layers in the stack even aggravate the situation. The effective fitting procedures minimizing the cost function, e.g., genetic algorithms or simulating annealing, also require tens of thousands single simulations to find a nonambiguous solution. In this case, the speeding up of calculations by using MEW plays essential role in software performance.

Calculation time is also important for simulation of diffuse x-ray scattering caused by rough interfaces in superlattices. Distorted-wave Born approximation used for calculation of diffuse scatter expresses the diffuse intensity $I(\alpha, \beta)$ through the matrix elements of potential $\hat{V}$ describing the rough interfaces and incident $\Psi_{i n}$ at angle $\alpha$ and reflected $\Psi_{\text {out }}$ at angle $\beta$ wave fields calculated for ideal SL (see, for example, Ref. 1):

$$
I(\alpha, \beta) \simeq\left|\left\langle\Psi_{\text {in }}(\alpha)|\hat{V}| \Psi_{\text {out }}(\beta)\right\rangle\right|^{2} .
$$

Each wave field contains the set of $2 N L$ amplitudes $T_{i}(\alpha)$, $R_{i}(\alpha)$ or $T_{i}(\beta), R_{i}(\beta)$, and the application of analytical formulas (21) instead of numerical solution of Parratt's equations reduces considerably the simulation time for calculation of diffusely scattered intensity in dependence on angles $\alpha$ and $\beta$.

The second seeming argument against the demand of MEW is that Eqs. (21) for amplitudes are valid for the case of ideal SL periodicity only. The superlattices with regularly (due to strain, for example) or irregularly (due to nonstability of growth conditions) fluctuating basic periods are quite a typical sample in nanocoating and semiconductor science and industry. Whereas the deformation of SL period can be taken into account directly by Parratt's equations, the averaging over the SL period fluctuations is complicated task. ${ }^{8}$ In the framework of MEW, this effect is taken into account by renormalization of the transfer matrix of basic period as, for example, the averaging of atomic vibrations in dynamical diffraction theory results in the Debye-Waller factor for x-ray susceptibility. The transfer matrix $\hat{M}^{(l)}$ and two-component vector $\mathbf{A}_{l}=\left(T_{l}, R_{l}\right)$ both depend on the period number $l$, therefore the recurrent formula containing these vectors in two successive SL periods can be introduced instead of Eq. (7) for eigenvalues.

$$
\hat{M}^{(l)} \mathbf{A}_{l}=\mathbf{A}_{(l+1)} .
$$

To introduce a parameter for description of abovementioned fluctuations, the transfer matrix $\hat{M}_{L}^{(l)}$ has to be reinterpreted on the basis of formal scattering theory. ${ }^{9}$ From this point of view, the matrix elements can be considered as elements of scattering operator $\hat{S}^{(l)}(z)$ defined with the functions of initial $i$ and final $f$ states

$$
\left(\hat{M}_{L}^{(l)}\right)_{i f}=(\hat{S})_{i f}=\int_{z_{l}}^{z_{l+1}} d z e^{-i k_{f}(z-l d)} \hat{S}_{L}(z-l d) e^{i k_{i}(z-l d)} .
$$


The matrix elements (24) are shown above to be independent on indices $l$, in the case ${ }^{10}$ of ideal superlattice. In real superlattices, the period thickness has a statistical fluctuations $\delta_{l}$, and thus $z_{l}=l d+\delta_{l}, \quad \delta_{l} \ll 1$. As a result, the transfer matrix obtains an additional random-phase coefficient

$$
\left(\hat{M}_{L}^{(l)}\right)_{i f}=\left(\hat{M}_{L}\right)_{i f} e^{i\left(k_{i}-k_{f}\right) \delta_{l}},
$$

which is absent in matrix $\hat{M}_{L}$, corresponding to ideal periodicity. The coherent part of the transfer matrix can be derived by averaging over the statistical distribution of random phases, in the same way as for coherent polarizability of crystal $^{7}$

$$
\begin{gathered}
\left(\hat{M}_{L}^{(l)}\right)_{i f}=\left\langle\left(\hat{M}_{L}\right)_{i f} e^{\left.i\left(k_{i}-k_{f}\right) \delta_{l}\right\rangle+\left(\hat{V}_{L}^{(l)}\right)_{i f} ;}\right. \\
\left(\hat{V}_{L}^{(l)}\right)_{i f} \equiv\left(\hat{M}_{L}^{(l)}\right)_{i f}-\left\langle\left(\hat{M}_{L}\right)_{i f} e^{i\left(k_{i}-k_{f}\right) \delta_{l}}\right\rangle .
\end{gathered}
$$

In particular case of negligible vertical correlation of interfacial roughness (which sometimes can be essential for interpretation of diffuse $\mathrm{x}$-ray scattering ${ }^{11}$ ), the coherent part of matrix elements in Eq. (26) obtains an additional factor, which does not depend on $l$. Assuming the Gaussian distribution of period fluctuations with root mean square (rms) $\sigma_{d}$, and averaging Eq. (26) over the fluctuations, we arrive at

$$
\left\langle\left(\hat{M}_{L}^{(l)}\right)_{i f}\right\rangle=e^{-1 / 2 \sigma_{d}^{2}\left(k_{f}-k_{i}\right)^{2}}\left(\hat{M}_{L}\right)_{i f} .
$$

The exponential factor reduces the amplitude of elastic scattering of the wave field by SL basic period analogously to Debye-Waller factor for crystallographic unit cell derived for x-ray polarizability of the crystal. ${ }^{7}$ This result causes the renormalization of matrix elements (18)

$$
\begin{array}{ll}
\left\langle M_{11}\right\rangle=M_{11}, & \left\langle M_{12}\right\rangle=M_{12} e^{-1 / 2 \sigma_{d}^{2}\left(k_{z L}+k_{z 1}\right)^{2}}, \\
\left\langle M_{22}\right\rangle=M_{22}, & \left\langle M_{21}\right\rangle=M_{21} e^{-1 / 2 \sigma_{d}^{2}\left(k_{z L}+k_{z 1}\right)^{2}} .
\end{array}
$$

Then the analytical solution of Eq. (23) for coherent wave is

$$
\mathbf{A}_{l}=\left(\lambda_{s}\right)^{l} \mathbf{A}^{(s)}
$$

with eigenvalues $\lambda_{s}$, determined from equation:

$$
\langle\hat{M}\rangle \mathbf{A}^{(s)}=\lambda_{s} \mathbf{A}^{(s)} .
$$

Figure 4 shows how the parameter $\sigma_{d}$, taking into account the fluctuation of SL period, influences the formation of the coherent SL peaks. The experimental measurements (dots) from superlattice $\left(\mathrm{W} / \mathrm{Al}_{2} \mathrm{O}_{3}\right)_{64}$ on the $\mathrm{Si}$ substrate with the nominal layer thicknesses (1.2/1.9) $64 \mathrm{~nm}$ have been taken by using Bruker D8 DISCOVER x-ray diffractometer in conventional $\theta-2 \theta$ geometry using knife edge collimator and antiscattering detector slit at $\mathrm{Cu} K_{\alpha}$ radiation. The first simulation (dashed) is carried out on the MEW basis for superlattice with constant periodicity and taking into account interface roughness by Nevot-Croce ${ }^{12}$ exponent (this curve is equivalent to Parratt's simulations)

$$
\bar{r}_{j+1, j}=r_{j+1, j} e^{-2 k_{j} k_{j+1} \sigma_{r}^{2}},
$$

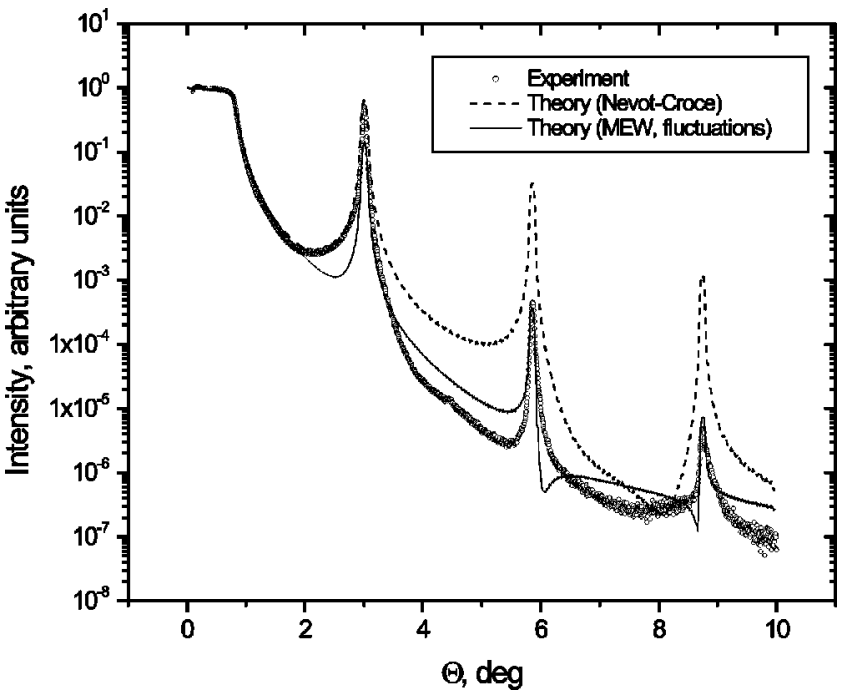

FIG. 4. Measured (dots) and simulated by MEW with NevotCroce exponent, corresponding to the rms of interface roughness $\sigma_{r}=0.25 \mathrm{~nm}$ (dashed line) and by MEW with the rms of period fluctuations $\sigma_{d}=0.25 \mathrm{~nm}$ (solid line) for x-ray reflectivities from $\left(\mathrm{W} / \mathrm{Al}_{2} \mathrm{O}_{3}\right)_{64}$ superlattice with layer thicknesses $(1.2 / 1.9)_{6} 4 \mathrm{~nm}$ on the Si substrate.

where the values $r_{j+1, j}$ are defined in Eq. (13) and parameter $\sigma_{r}$ is the rms of interface roughness.

In general case, the imperfection of interfaces described by $\sigma_{r}$ is not reduced to the fluctuation of layers thicknesses, defined by parameter $\sigma_{d}$. The interface roughness can be approached by thickness fluctuation only in the case of rather large in-plane correlation length. Essentially different impact of both parameters on the fitting of experimental data is demonstrated in Fig. 4. The value $\sigma_{r}$ has been roughly fitted as $0.25 \mathrm{~nm}$ and equal for all interfaces, and the reflectivity has been convoluted with resolution function describing the limited angular resolution of detector. The second simulation (solid line) is done by using MEW with integral SL period fluctuations [Eq. (27)] with the same $\sigma_{d}=0.25 \mathrm{~nm}$. In both cases, the same roughness rms $\sigma_{r}$ and $\sigma_{d}$ influences the amplitude of SL peaks in different way, however, the latter approach explains the experiment better. It should be noted that Fig. 4 represents only the qualitative fit; the influence of incoherent matrix $\left(\hat{V}_{L}^{(l)}\right)_{i f}$ in recurrent Eq. (23) on the phase of the transfer matrix seems to have been taken into account to reach better fit on the wings of SL peaks. However, this study is out of the scope of the present work.

\section{MEW FOR HIGH-RESOLUTION X-RAY DIFFRACTION FROM SUPERLATTICES}

The superlattices consisting of crystalline layers are widely used in modern semiconductor industry, and highresolution x-ray diffraction is one of the most effective techniques for their investigations. The parameters characterizing the sample, e.g., layer thicknesses, doping concentrations, lattice deformation, lattice mismatch and others, are obtained 


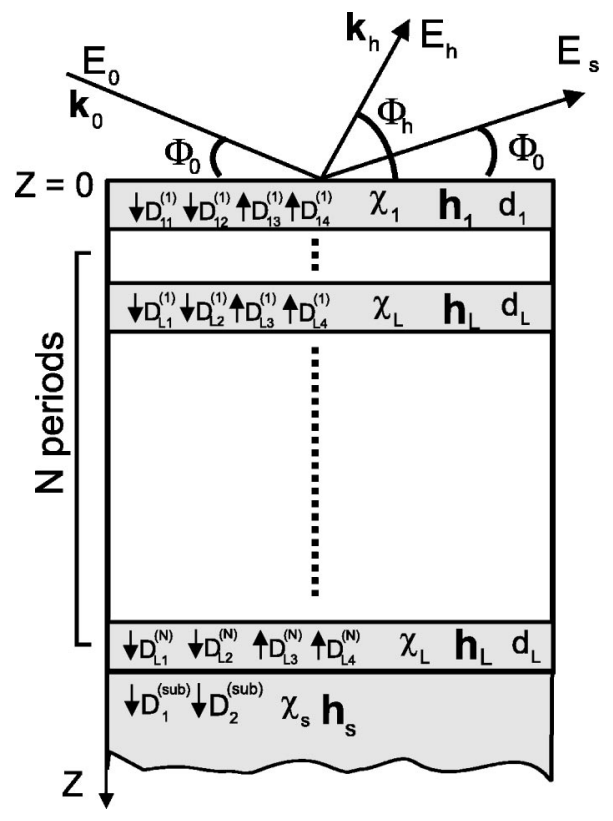

FIG. 5. Sketch of the wave fields for crystalline superlattice.

from $\mathrm{x}$-ray measurements in various experimental geometries, including extremely asymmetrical and grazingincidence diffraction. Theoretical interpretation of these data requires a considerable computer resources that makes an optimization of calculation algorithm for HRXRD very actual problem. In the same way as for X-ray reflectivity, the application of MEW for high-resolution x-ray diffraction is based on the analytical calculation of interference between the waves scattered from equivalent layers of superlattice period. However, the $\mathrm{x}$-ray scattering from crystalline structure involves more complicated than in reflectometry transfer matrices, which possess a higher rank in this case.

The theory presented below assumes that the superlattice consists of repeating $N$ times basic SL periods, composed of $L$ monocrystal layers. The difference in crystallographic structure of these layers can be caused either by different elemental composition of layers or gradiental lattice deformation of single layer due to the external forces. There are few ways to calculate the transfer matrix $\hat{M}_{L}$ for x-ray diffraction from the crystalline layers. Takagi-Taupin ${ }^{13}$ formalism, being relatively simple in realization, gives a solution in approximation of slow variation of crystalline structure of layers stack. In opposite, the matrix formalism ${ }^{14}$ of dynamical diffraction theory delivers an exact solution for transfer matrix $\hat{M}_{L}$, and here we use this approach along with the notations adopted in Ref. 14. The wave field with certain polarization $(\sigma$ or $\pi$ ) inside the layer $n$ is described by the wave field

$$
D_{n}(\mathbf{r})=e^{i \mathbf{k}_{n} \mathbf{r}}\left[D_{0 n}+D_{h n} e^{i \mathbf{h}_{n} \mathbf{r}}\right]
$$

here $\mathbf{k}_{n}$ and $\mathbf{k}_{h n}=\mathbf{k}_{n}+\mathbf{h}_{n}$ are the wave vectors of transmitted and diffracted by reciprocal lattice vector $\mathbf{h}_{n}$ waves in $n$ layer. The notations used below are shown in Fig. 5. The

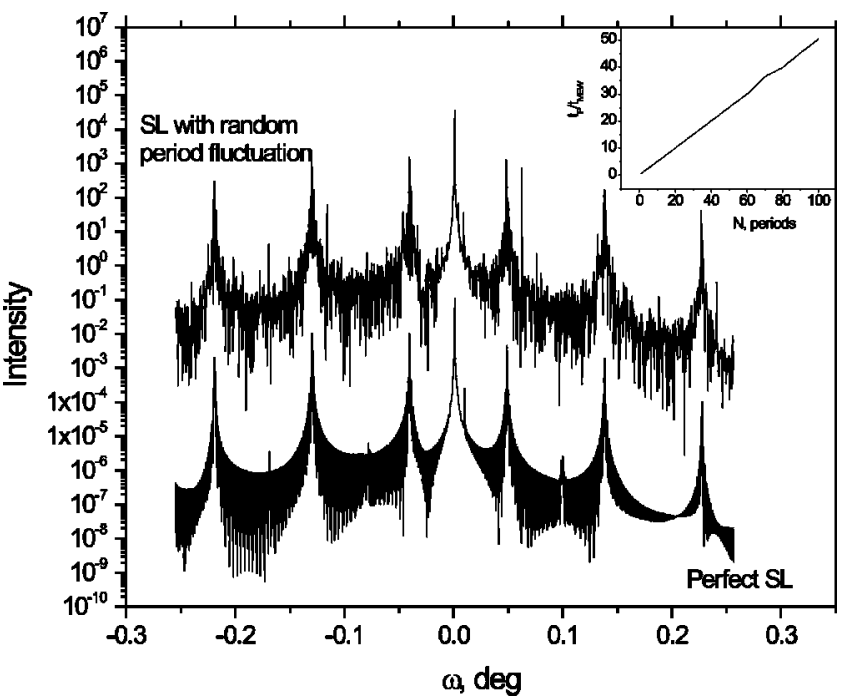

FIG. 6. The simulations of x-ray diffraction from $\left(\mathrm{Ge} / \mathrm{Si} / \mathrm{Si}_{0.8} \mathrm{Ge}_{0.2}\right)_{100}$ perfect superlattice on the Si substrate both by the recursive method and MEW are indistinguishable (lower curve). Inset shows the ratio of the computer times required for simulation by recursive method $\left(t_{R}\right)$ and MEW $\left(t_{M E W}\right)$ as the function of SL periods number. Upper curve shifted vertically for clarity by a factor of $10^{5}$ shows the simulated x-ray diffraction from the same superlattice with randomly fluctuating basic SL period, $\sigma_{L}$ $=1 \mathrm{~nm}$.

amplitudes $D_{0 n}, D_{h n}$ are assumed to be constant within the layer and satisfy to the equations of dynamical diffraction theory

$$
\begin{aligned}
& \left(k_{n}^{2}-k^{2}\right) D_{0 n}=k_{n}^{2}\left[\chi_{0}^{n} D_{0 n}+\chi_{-h}^{n} D_{h n}\right], \\
& \left(k_{h n}^{2}-k^{2}\right) D_{h n}=k_{n}^{2}\left[\chi_{0}^{n} D_{h n}+\chi_{h}^{n} D_{0 n}\right],
\end{aligned}
$$

with Fourier components $\chi_{0}^{n}, \chi_{h}^{n}$ of x-ray polarizability for $n$ layer. Using the notations of Fig. 6 for vector components

$$
k_{z}=k \gamma_{0}, \quad k_{h z}=k \gamma_{h}, \quad h_{z n}=k \psi_{n}, \quad k_{z n}=k u_{n},
$$

the parameter $u_{n}$, determining the effective refraction index for $\mathrm{x}$-ray diffraction, follows from the equation: ${ }^{14}$

$$
\begin{gathered}
\left(u_{n}^{2}-\gamma_{0}^{2}-\chi_{0}^{n}\right)\left[\left(u_{n}+\psi_{n}\right)^{2}-\gamma_{h}^{2}-\chi_{0}^{n}\right]-\chi_{-h}^{n} \chi_{h}^{n}=0, \\
\gamma_{h}^{2}=\left(\gamma_{0}+\psi\right)^{2}+\alpha .
\end{gathered}
$$

The variable $\alpha$ defines a deviation of the vector $\mathbf{k}$ from the exact Bragg condition at the top layer interface of basic superlattice period, and the amplitudes of transmitted and diffracted waves are connected by expressions

$$
D_{h n}^{j}=v_{n}^{j} D_{0 n}^{j}, \quad v_{n}^{j}=\left[\left(u_{n}^{j}\right)^{2}-\gamma_{0}^{2}-\chi_{0}^{n}\right] / \chi_{-h}^{n} .
$$

The evolution of wave field inside the basic period of superlattice is determined by the system of equations for amplitudes $D_{0 n}^{j}$, which follows from the continuity condition for the wave fields at the boundaries of layers (interfaces). 
The solution for this equation system for arbitrary experimental geometry is represented ${ }^{4}$ by product of $2 L(4 \times 4)$ matrices $\hat{S}_{n}$ and $\hat{F}_{n}$ :

$$
\begin{gathered}
\hat{S}_{0}=\left(\begin{array}{cccc}
1 & 0 & 1 & 0 \\
0 & 1 & 0 & 1 \\
\gamma_{0} & 0 & -\gamma_{0} & 0 \\
0 & \gamma_{h} & 0 & -\gamma_{h}
\end{array}\right), \\
\hat{S}_{n}=\left(\begin{array}{cccc}
1 & 1 & 1 & 1 \\
v_{n}^{1} & v_{n}^{2} & v_{n}^{3} & v_{n}^{4} \\
u_{n}^{1} & u_{n}^{2} & u_{n}^{3} & u_{n}^{4} \\
w_{n}^{1} & w_{n}^{2} & w_{n}^{3} & w_{n}^{4}
\end{array}\right), \\
\left(\hat{F}_{n}\right)_{i j}=\delta_{i j} \exp \left[i k u_{n}^{j}\left(z_{n+1}-z_{n}\right)\right], \quad w_{n}^{j}=v_{n}^{j}\left(u_{n}^{j}+\psi_{n}\right) .
\end{gathered}
$$

In conventional dynamical diffraction theory, the approximate solution of dispersion equation, Eq. (31), is used, which is reduced in this case to the second-order equation, and the matrices $S_{n}$ have a dimension $(2 \times 2)$. The exact solution of dispersion equation and complete matrices (33) have to be used for the grazing-incidence (-exit) geometry, ${ }^{14}$ and for the diffraction from superlattices with short period, when satellite peaks are located far from the exact Bragg condition. ${ }^{15}$ The numerical problems arise for superlattices with large number of periods $N$, where multiple production of $(4 \times 4)$ matrices has to be done. ${ }^{14}$ These problems are caused by operations with matrices, the elements of which grow exponentially with the increasing value of $N$. In order to avoid these difficulties, new algorithm was suggested in Ref. 14 when the matrices are divided into the blocks of $(2 \times 2)$. The four-wave amplitudes $D_{0 n}^{j}$ being combined into two twocomponent vectors $\bar{T}_{n}=\left(D_{0 n}^{1} ; D_{0 n}^{2}\right), \bar{R}_{n}=\left(D_{0 n}^{1} ; D_{0 n}^{2}\right)$ are then related by the equations analogous to Parratt's ones. ${ }^{14}$ This algorithm provides the required accuracy for arbitrary number of superlattice periods, however, increases twice the computation time in comparison with conventional matrix method. The MEW is shown to improve the accuracy as well as reduce the time of calculation.

To construct the transition matrix $\hat{M}_{L}$ for basic period, four components of wave field in $n$th layer are denoted as the four-vector $\bar{D}_{n}$. The spacial phase of wave field amplitudes in every layer has to be equal at the entrance interface of layer. Using the matrices (33), the boundary conditions for amplitudes in basic period are written as a system of $L$ vector equations

$$
\hat{S}_{1} \hat{F}_{1} \bar{D}_{1}=\hat{S}_{2} \bar{D}_{2}, \ldots, \quad \hat{S}_{L} \hat{F}_{L} \bar{D}_{1}=\hat{S}_{1} \bar{D}_{L+1} .
$$

Then the matrix $\hat{M}_{L}$ follows straightforward from Eqs. (34)

$$
\hat{M}_{L}=\hat{X}_{L} \hat{X}_{L-1} \ldots \hat{X}_{1}, \quad \hat{X}_{k}=\hat{S}_{k+1}^{-1} \hat{S}_{k} \hat{F}_{k} .
$$

Let us now introduce the normalized four-components eigenvectors $\bar{\Psi}^{s}$ and eigenvalues $\lambda_{s}$, enumerated in natural order of solutions for the equation

$$
\begin{gathered}
\hat{M}_{L} \bar{\Psi}^{s}=\lambda_{s} \bar{\Psi}^{s}, \quad s=1, \ldots, 4, \quad \sum_{i=1}^{4}\left(\Psi_{i}^{s}\right)^{*}\left(\Psi_{i}^{s}\right)=1, \\
\left|\lambda_{1}\right|<\left|\lambda_{2}\right|<\left|\lambda_{3}\right|<\left|\lambda_{4}\right| .
\end{gathered}
$$

The matrix $\hat{M}_{L}$ is not self-conjugated due to the absorption in the crystals, and therefore its eigenvectors do not satisfy the conditions of completeness and orthogonality, making the eigenvalues complex valued. The MEW technique using these eigenwaves is realized in the same way as in Eq. (31). First, the wave field in the topmost layer of the basic period has to be represented as a linear superposition of the eigenvectors with coefficients defined by continuity of the vacuum wave field vector $\bar{E}=\left(1,0, E_{r}, E_{h}\right)$ at the sample surface

$$
\bar{D}_{1}^{(1)}=\sum_{s=1}^{4} A_{s} \bar{\Psi}^{s}, \quad \hat{S}_{0} \bar{E}=\hat{S}_{1} \bar{D}_{1}^{(1)}=\hat{S}_{1} \sum_{s=1}^{4} A_{s} \bar{\Psi}^{s}
$$

The evolution of wave field through $N$ periods of superlattice is calculated algebraically

$$
\bar{D}_{1}^{(N+1)}=(\hat{M})^{N} \bar{D}_{1}^{(1)}=\sum_{s=1}^{4}\left(\lambda_{s}\right)^{N} A_{s} \bar{\Psi}^{s} .
$$

The wave field of x-ray beam in substrate is defined by fourvector $\bar{D}=\left(D_{1}^{s u b}, D_{2}^{\text {sub }}, 0,0\right)$. The amplitudes of the waves reflected from the bottom interface of the substrate are assumed to be zero due to the damping out of wave fields within the thick sample, $E_{r}$ and $E_{h}$ define the amplitudes of reflected and diffracted waves in vacuum. ${ }^{14}$

The conditions of continuity at the interface between superlattice and substrate has to be applied to the four-vector $\bar{D}_{L}^{(N)}$, determining the wave field amplitude in the bottommost layer of the stack (Fig. 5)

$$
\bar{D}_{L}^{(N)}=\hat{F}_{L}^{-1} \hat{S}_{L}^{-1} \hat{S}_{1} \bar{D}_{1}^{(N+1)}, \quad \hat{S}_{\text {sub }} \bar{D}=\hat{S}_{L} \hat{F}_{L} \bar{D}_{L}^{(N)} .
$$

As a result, the system of equations for eight unknown values $\left(E_{r}, E_{h}, A_{s}, D_{1,2}^{s u b}\right)$ can be written as

$$
\begin{gathered}
\left(\hat{S}_{1}^{-1} \hat{S}_{\text {sub }}\right)_{i j} D_{j}^{s u b}=\sum_{s=1}^{4}\left(\lambda_{s}\right)^{N} A_{s} \Psi_{i}^{s}, \\
\left(\hat{S}_{1}^{-1} \hat{S}_{0}\right)_{i j} E_{j}=\sum_{s=1}^{4} A_{s} \Psi_{i}^{s} .
\end{gathered}
$$

The orthogonality condition for eigenvectors (36) cannot be used for solution of these equations [see discussion after 
Eq. (36)]. However, this system can be solved in general form, if four components of four eigenvectors are considered as $(4 \times 4)$ matrix

$$
\Psi_{i}^{s} \rightarrow(\hat{\Psi})_{i}^{s} .
$$

Then the values $A_{s}$ can be excluded from Eqs. (40) using the reverse matrix $\hat{\Psi}^{-1}$

$$
\left(\hat{S}_{1}^{-1} \hat{S}_{0}\right)_{i j} E_{j}=\sum_{s=1}^{4}\left(\lambda_{s}\right)^{-N}(\hat{\Psi})_{i}^{s}\left(\hat{\Psi}^{-1}\right)_{j}^{s}\left(\hat{S}_{1}^{-1} \hat{S}_{s u b}\right)_{j k} D_{k}^{s u b} .
$$

In order to avoid the exponentially increasing terms, the normalized matrix can be defined

$$
(\hat{Q})_{i j}=\sum_{s=1}^{4}\left(\frac{\lambda_{1}}{\lambda_{s}}\right)^{N}(\hat{\Psi})_{i}^{s}\left(\hat{\Psi}^{-1}\right)_{j}^{s},
$$

and Eq. (41) is written then in the following form

$$
\bar{E}=\frac{1}{\lambda_{1}^{N}} \hat{Z} \bar{D}^{s u b}, \quad \hat{Z}=\hat{S}_{0}^{-1} \hat{S}_{1} \hat{Q} \hat{S}_{1}^{-1} \hat{S}_{s u b}
$$

and the exponentially increasing value $\left(\lambda_{1}\right)^{-N}$ is canceled in expression for amplitude $E_{h}$,

$$
E_{h}=\frac{Z_{22} Z_{41}-Z_{42} Z_{21}}{Z_{11} Z_{22}-Z_{12} Z_{21}}
$$

Thus, the following problems of $\mathrm{x}$-ray diffraction simulation are solved on the basis of MEW: (i) the time of calculation depends no longer on repetition period $N$, (ii) numerilcal algorithm operates with only finite values. Figure 6 shows the spectrum for the superlattice with crystalline layers $\left(\mathrm{Ge} / \mathrm{Si} / \mathrm{Si}_{0.8} \mathrm{Ge}_{0.2}\right)_{100}$ with the thicknesses $(30 / 20 / 10)$ $\mathrm{nm}$, respectively, on the $\mathrm{Si}$ substrate. The curve has been simulated both by the recursive method ${ }^{14}$ and MEW. Evidently, the results are undistinguishable because both methods are exact. However, MEW decreases drastically the calculation time $t_{M}$ in comparison with time $t_{R}$, required for recursive method, especially for multiperiodical superlattices (inset on Fig. 6). Method of eigenwaves permits to naturally introduce an important integral characteristics of superlattices, viz. rms fluctuation $\sigma_{L}$ of basic period. The fluctuations are usually caused by imperfections of interfaces or/and by statistical fluctuations of sample growth conditions (temperature, etc.). Similarly to reflectivity case (27), MEW takes into account period fluctuations by Debye-Waller factor in nondiagonal elements of averaged transition matrix $\left\langle\hat{M}_{L}\right\rangle$ :

$$
\left\langle\left(\hat{M}_{L}\right)_{i j}\right\rangle=\left(\hat{M}_{L}\right)_{i j} e^{-1 / 2 \sigma_{L}^{2} k^{2}\left(u_{1}^{i}-u_{L}^{j}\right)^{2}}
$$

with the solutions $u_{n}^{i}$ of Eq. (31).

The period fluctuations result in the reshaping of SL peaks in the same way as it was described in Sec. III for reflectometry. Upper curve in the Fig. 6 shows the simulated x-ray diffraction from the same superlattice with period fluctuation $\sigma_{L}=1 \mathrm{~nm}$. The introduction of this parameter may be essential for the fitting accuracy in some cases. The structure of SL peaks is formed due to the interference of waves, scattered from one-dimensional periodical layers stack, and therefore the positions of peaks are defined by averaged SL period and do not depend on period fluctuation. ${ }^{16}$ Simultaneously, if the nondiagonal matrix elements (45) are taken into account, the spectral width of the peaks changes as well as the integral photon number in the peaks. Therefore, the success of the fitting of experimental measurements from superlattice with fluctuating period essentially depends on convolution of theoretical intensity with instrumental function of detector, however, this problem is out of the scope of present work.

\section{CONCLUSIONS}

The effective method for simulation of x-ray intensity scattered from the periodical superlattices is described. The essential advantage of proposed technique is independence of calculation time on the total number of repetition periods of SL. This permits to speed up the sample parameter fitting procedure (trial-and-error methods) used for experimental data treatment in modern nanotechnology. The method also delivers the analytical expression for wave fields in all the layers of multilayered structure without solving of recurrent equations. This is essential in the cases when wave fields are used for calculation of matrix elements of perturbation operator. For example, these wave fields can be used for simulation of diffusely scattered x-ray intensity by means of distorted-wave Born approximation, ${ }^{1}$ or for calculation of $\mathrm{x}$-ray radiation spectra from electrons. ${ }^{17}$

\section{ACKNOWLEDGMENTS}

The authors thank M. Lyubchenko (Bruker AXS) for measurements. This work is supported by Bruker AXS GmbH and International Science and Technology Center (Grant No. B-626). The authors thank M. Lyubchenko (Bruker AXS) for measurements.

\footnotetext{
${ }^{1}$ U. Pietsch, V. Holy, and T. Baumbach, High Resolution X-Ray Scattering from Thin Films and Multilayers (Springer Verlag, Heidelberg, 1999).

${ }^{2}$ L.G. Parratt, Phys. Rev. 95, 359 (1954).

${ }^{3}$ L. Abeles, Ann. Phys. (Paris) 3, 504 (1948); 5, 596 (1950).
}

\footnotetext{
${ }^{4}$ S.A. Stepanov and R. Köhler, J. Phys. D 27, 1923 (1994).

${ }^{5}$ D.W. Berremen, Phys. Rev. B 14, 4313 (1976).

${ }^{6}$ A.M. Dikhne, Zh. Eksp. Teor. Fiz. 40, 1423 (1961).

${ }^{7}$ R. W. James, The Optical Principles of the Diffraction of X-Rays (Ox Bow Press, Woodbridge, 1962).
} 
${ }^{8}$ A.P. Payne and B.M. Clemens, Phys. Rev. B 47, 2289 (1993).

${ }^{9}$ T.-Y. Wu and T. Omura, Quantum Theory of Scattering (PrenticeHall, New York, 1962).

${ }^{10}$ S.K. Sinha, E.B. Sirota, S. Garoff, and H.B. Stanley, Phys. Rev. B 38, 2297 (1988).

${ }^{11}$ V. Holy and T. Baumbach, Phys. Rev. B 49, 10668 (1994).

${ }^{12}$ L. Nevot and P. Croce, Rev. Phys. Appl. 15, 761 (1980).

${ }^{13}$ S. Takagi, Acta Crystallogr. 15, 1311 (1962); D. Taupin, Bull. Soc. Fr. Mineral. Cristallogr. 87, 469 (1964).
${ }^{14}$ S.A. Stepanov, E.A. Kondrashkina, R. Köhler, D.V. Novikov, G. Materlik, and S.M. Durbin, Phys. Rev. B 57, 4829 (1998).

${ }^{15}$ L. De Caro, C. Giannini, and L. Tapfer, Phys. Rev. B 56, 9744 (1997).

${ }^{16}$ L. D. Landau and E. M. Lifshitz, Electrodynamics of the Condensed Media (Nauka, Moscow, 1982).

${ }^{17}$ I.D. Feranchuk and A. Ulyanenkov, Phys. Rev. B 63, 155318 (2001). 